\title{
Age Problem in the Holographic Dark Energy Model
}

\author{
Hao Wei* \\ Department of Physics and Tsinghua Center for Astrophysics, \\ Tsinghua University, Beijing 100084, China \\ Shuang Nan Zhang \\ Department of Physics and Tsinghua Center for Astrophysics, \\ Tsinghua University, Beijing 100084, China \\ Key Laboratory of Particle Astrophysics, Institute of High Energy Physics, \\ Chinese Academy of Sciences, Beijing 100049, China \\ Physics Department, University of Alabama in Huntsville, Huntsville, AL 35899, USA
}

\begin{abstract}
In this note, we test the original holographic dark energy model with some old high redshift objects. The main idea is very simple: the universe cannot be younger than its constituents. We find that the original holographic dark energy model can be ruled out, unless a lower Hubble constant is taken.

PACS numbers: 95.36.+x, 98.80.Es, 98.80.-k
\end{abstract}

* email address: haowei@mail.tsinghua.edu.cn 


\section{INTRODUCTION}

Dark energy [1] has been one of the most active fields in modern cosmology since the discovery of accelerated expansion of our universe [2, 3, 4, 5, 6, 7, 8, 9]. The simplest candidate of dark energy is a tiny positive cosmological constant. However, as is well known, it is plagued with the so-called "cosmological constant problem" and "coincidence problem" 1]. Many dynamical dark energy models have been proposed, such as quintessence [10, 11], phantom [12, 13, 14], k-essence [15], quintom [16, 17, [18, 19], hessence [20], and so on.

The so-called holographic dark energy is now an interesting candidate of dark energy, which has been studied extensively in the literature. It is proposed from the holographic principle [21, 22] in the string theory. For a quantum gravity system, the local quantum field cannot contain too many degrees of freedom, otherwise the formation of black hole is inevitable and then the quantum field theory breaks down. In the thermodynamics of the black hole [23, 24], there is a maximum entropy in a box of size $L$, namely the so-called Bekenstein entropy bound $S_{B H}$, which scales as the area of the box $\sim L^{2}$, rather than the volume $\sim L^{3}$. To avoid the breakdown of the local quantum field theory, Cohen et al. [25] proposed a more restrictive bound, i.e. the energy bound. If $\rho_{\Lambda}$ is the quantum zero-point energy density caused by a short distance cut-off, the total energy in a box of size $L$ cannot exceed the mass of a black hole of the same size [25], namely $L^{3} \rho_{\Lambda} \lesssim L M_{p l}^{2}$, where $M_{p l} \equiv(8 \pi G)^{-1 / 2}$ is the reduced Planck mass. The largest IR cut-off $L$ is the one saturating the inequality. Thus,

$$
\rho_{\Lambda}=3 c^{2} M_{p l}^{2} L^{-2}
$$

where the numerical constant $3 c^{2}$ is introduced for convenience. If we choose $L$ as the size of the universe, for instance the Hubble scale $H^{-1}$, the resulting $\rho_{\Lambda}$ is comparable to the observational dark energy [26, 27]. However, Hsu [27] pointed out that in this case the resulting equation-of-state parameter (EoS) is equal to zero, which cannot accelerate the expansion of our universe. The other possibility [28] is to choose $L$ as the particle horizon

$$
R_{H}=a \int_{0}^{t} \frac{d \tilde{t}}{a}=a \int_{0}^{a} \frac{d \tilde{a}}{H \tilde{a}^{2}},
$$

where $H \equiv \dot{a} / a$ is the Hubble parameter; $a$ is the scale factor of the universe; a dot denotes the derivative with respect to cosmic time $t$. However, it is easy to find that in this case the EoS is always larger than $-1 / 3$ and also cannot accelerate the expansion of our universe [29]. To get an accelerating universe, Li proposed in [29] to choose $L$ as the future event horizon

$$
R_{h}=a \int_{t}^{\infty} \frac{d \tilde{t}}{a}=a \int_{a}^{\infty} \frac{d \tilde{a}}{H \tilde{a}^{2}} .
$$

In this case, the EoS of the holographic dark energy can be less than $-1 / 3[29]$.

The theoretical aspects of the holographic dark energy have been studied extensively in the literature, see [30, 31, 32, 33, 34, 35, 36, 37, 73, 74, 75, 76] for examples. Also, the holographic dark energy has been tested and constrained by various observations, such as SNe Ia [38], CMB 39], X-ray gas mass fraction of galaxy clusters [40], the differential ages of passively evolving galaxies [41], Sandage-Leob test [42], and so on. In particular, the holographic dark energy has been constrained recently by combining the latest SNe Ia, galaxy clustering and CMB anisotropy [43]. In the previous works, to our knowledge, the holographic dark energy is consistent with current observational data.

In this note, we test the original holographic dark energy with some old high redshift objects (OHRO). The main idea is very simple: the universe cannot be younger than its constituents. In history, the age problem played an important role in the cosmology for many times. For example [44], before the discovery of accelerated expansion of our universe, many people believed that we are living in a matter-dominated spatially flat Friedmann-Robertson-Walker (FRW) universe. However, it is found that in this case, the present age of the universe, $t_{0}=(2 / 3) H_{0}^{-1}$ [45], is smaller than the ages inferred from old globular clusters. The matter-dominated flat FRW universe is ruled out unless $h<0.48$, where $h$ is defined by the Hubble constant $H_{0}=100 \mathrm{hkm} / \mathrm{s} / \mathrm{Mpc}$. Since the age of the universe for the matter-dominated closed 
model is even smaller than the flat case 45], the age problem remains. Thus, for the matter-dominated FRW models without cosmological constant, only extremely open universe may be old enough to solve the age problem [44]. The age problem becomes even more serious when we consider the age of the universe at high redshift (rather than at present day, $z=0$ ). By now, there are some OHRO are discovered, for instance, the 3.5 Gyr old galaxy LBDS 53W091 at redshift $z=1.55$ [46, 47], the 4.0 Gyr old galaxy LBDS 53W069 at redshift $z=1.43$ [48]. In addition, the old quasar APM 08279+5255 at $z=3.91$ [51, 52 is also used extensively. Its age is estimated to be 2.0-3.0 Gyr [51, 52]. In [53], by using a different method, its age is reevaluated to be $2.1 \mathrm{Gyr}$. To assure the robustness of our analysis, we use the most conservative lower age estimate 2.0 Gyr for the old quasar APM $08279+5255$ at $z=3.91$ throughout this work. In history, the former two old galaxies at $z=1.43,1.55$ have been shown to be incompatible with the age estimate for a flat FRW universe without cosmological constant. Combining with other independent observations, it is suggested that the more realistic model is the flat FRW universe with cosmological constant, i.e., the $\Lambda$ CDM model. In the $\Lambda$ CDM model, a period of cosmic acceleration at low redshift is allowed, and then the universe can have a larger age than the one of matter-dominated model. Therefore, the old galaxies at high redshift can be accommodated.

In fact, these three OHRO at $z=1.43,1.55$ and 3.91 have been used to test many dark energy models, such as the $\Lambda$ CDM model [44, 53, 54], the dark energy models with different EoS parameterizations [49, 50], the generalized Chaplygin gas [55], the $\Lambda(t) \mathrm{CDM}$ model [56], the model-independent EoS of dark energy [57], the scalar-tensor quintessence [58], the $f(R)=\sqrt{R^{2}-R_{0}^{2}}$ model [59], the DGP braneworld model [60, 61], the power-law parameterized quintessence model [62], and so on. It is found that the two OHRO at $z=1.43$ and 1.55 can be easily accommodated in most dark energy models, whereas the OHRO at $z=3.91$ can not, even in the $\Lambda$ CDM model. These results give rise to the new age crisis in the dark energy models.

In this note, we consider the age problem in the original holographic dark energy model. In the next section, after a brief review of the holographic dark energy model, we compare the ages of these three OHRO with the ones estimated from the holographic dark energy model with the parameters constrained by the latest SNe Ia, galaxy clustering and CMB anisotropy [43]. We find that the two OHRO at $z=1.43$ and 1.55 can be accommodated in the original holographic dark energy model, whereas the OHRO at $z=3.91$ can not. We then examine this age problem from another perspective. Following [50], we keep the model parameters $c$ and $\Omega_{m 0}$ free, and find out the parameter space which can accommodate these three OHRO by means of plotting the contours of the dimensionless age parameter. The allowed parameter space can be ruled out at $1 \sigma$ by the WMAP three-year (WMAP3) bound $\Omega_{m 0}=0.268 \pm 0.018$ [6] . Even when we use the loosest and model-independent cluster estimate $\Omega_{m 0}=0.3 \pm 0.1$ [63], the allowed parameter space becomes very narrow and can also be ruled out by the combined constraints from the latest SNe Ia, galaxy clustering and CMB anisotropy [43]. To alleviate the age problem in the holographic dark energy model, as shown in Sec. III a lower Hubble constant is needed, such as the one recently advocated by Sandage and collaborators. In the last section, some concluding remarks are given.

\section{THE HOLOGRAPHIC DARK ENERGY MODEL VERSUS OHRO}

The age of our universe at redshift $z$ is given by [44, 45, 49, 50, 53, 54, 55]

$$
t(z)=\int_{z}^{\infty} \frac{d \tilde{z}}{(1+\tilde{z}) H(\tilde{z})}
$$

It is convenient to introduce the so-called dimensionless age parameter [50]

$$
T_{z}(z) \equiv H_{0} t(z)=\int_{z}^{\infty} \frac{d \tilde{z}}{(1+\tilde{z}) E(\tilde{z})}
$$

where $E(z) \equiv H(z) / H_{0}$. At any redshift, the age of our universe should be larger than, at least equal to, the age of the OHRO, namely

$$
T_{z}(z) \geq T_{o b j} \equiv H_{0} t_{o b j}, \quad \text { or equivalently, } \quad \tau(z) \equiv T_{z}(z) / T_{o b j} \geq 1
$$


where $t_{o b j}$ is the age of the OHRO. We consider a flat FRW universe which contains the holographic dark energy and pressureless matter. The Friedmann equation is given by

$$
3 M_{p l}^{2} H^{2}=\rho_{m}+\rho_{\Lambda},
$$

where $\rho_{m}$ and $\rho_{\Lambda}$ are the energy density of the pressureless matter and the holographic dark energy respectively. Thus, it is easy to find that

$$
E(z)=\left[\frac{\Omega_{m 0}(1+z)^{3}}{1-\Omega_{\Lambda}}\right]^{1 / 2},
$$

where $\Omega_{i} \equiv \rho_{i} /\left(3 M_{p l}^{2} H^{2}\right)$ is the fractional energy density; the subscript " 0 " indicates the present value of the corresponding quantity; $a=(1+z)^{-1}$ (we set $a_{0}=1$ ). Following [29, 43], from Eq. (11) and replacing $L$ with the future event horizon $R_{h}$ in Eq. (3), we have

$$
\int_{a}^{\infty} \frac{d \ln \tilde{a}}{H \tilde{a}}=\frac{c}{H a \sqrt{\Omega_{\Lambda}}} .
$$

From Eq. (8), we obtain

$$
\frac{1}{H a}=\sqrt{a\left(1-\Omega_{\Lambda}\right)} \frac{1}{H_{0} \sqrt{\Omega_{m 0}}} .
$$

Substituting it into Eq. (9), we find that

$$
\int_{x}^{\infty} e^{\tilde{x} / 2} \sqrt{1-\Omega_{\Lambda}} d \tilde{x}=c e^{x / 2} \sqrt{\frac{1}{\Omega_{\Lambda}}-1},
$$

where $x \equiv \ln a$. Taking derivative with respect to $x$ in both side of Eq. (11) and noting that $a=(1+z)^{-1}$, we finally obtain that $[29,43]$

$$
\frac{d \Omega_{\Lambda}}{d z}=-(1+z)^{-1} \Omega_{\Lambda}\left(1-\Omega_{\Lambda}\right)\left(1+\frac{2}{c} \sqrt{\Omega_{\Lambda}}\right) .
$$

One can get $\Omega_{\Lambda}(z)$ by solving this differential equation with the initial condition $\Omega_{\Lambda 0}=1-\Omega_{m 0}$. Substituting the $\Omega_{\Lambda}(z)$ into Eqs. (8) and (5), the dimensionless age parameter of our universe $T_{z}(z)$ is in hand. Then, we can compare $T_{z}(z)$ with the $T_{o b j}$ of the three OHRO. It is worth noting that from Eqs. (12), (8) and (5), $T_{z}(z)$ is independent of the Hubble constant $H_{0}$. On the other hand, $T_{o b j}$ is proportional to the Hubble constant $H_{0}$. The lower $H_{0}$, the smaller $T_{o b j}$ is.

\begin{tabular}{c|c|c|c}
\hline \hline$\left(c, \Omega_{m 0}\right)$ & $\tau(3.91)$ & $\tau(1.43)$ & $\tau(1.55)$ \\
\hline$(0.73,0.26)$ & 0.918 & 1.276 & 1.362 \\
$(1.17,0.26)$ & 0.906 & 1.234 & 1.320 \\
$(0.73,0.32)$ & 0.829 & 1.158 & 1.236 \\
$(1.17,0.32)$ & 0.820 & 1.128 & 1.205 \\
\hline \hline
\end{tabular}

TABLE I: The ratio $\tau(z) \equiv T_{z}(z) / T_{o b j}$ at $z=3.91,1.43$ and 1.55 , for different model parameters $c$ and $\Omega_{m 0}$, in the case of SNIa+CMB+LSS without prior on $h$.

In [43], the original holographic dark energy has been constrained recently by combining the latest SNe Ia, galaxy clustering and CMB anisotropy. In the case of SNIa+CMB+LSS without prior on $h$, the fit values are $c=0.91_{-0.18}^{+0.26}$ and $\Omega_{m 0}=0.29_{-0.03}^{+0.03}$, while the best fit value of $h$ is 0.63 . In the case of 
SNIa + CMB + LSS with prior $h=0.72 \pm 0.08$ (which is the final result of Freedman et al. 64 ]), the fit values are $c=0.91_{-0.19}^{+0.23}$ and $\Omega_{m 0}=0.29 \pm 0.03$. In the case of SNIa+CMB+LSS with prior $0.64 \leq h \leq 0.80$, the fit values are $c=0.82_{-0.13}^{+0.11}$ and $\Omega_{m 0}=0.28_{-0.02}^{+0.03}$. We will examine the age problem in these three cases one by one. In the case of SNIa+CMB+LSS without prior on $h$, the dimensionless age parameter of the OHRO at $z=3.91,1.43$ and 1.55 are $T_{o b j}=0.129,0.256$ and 0.226 respectively, for the best fit $h=0.63$. In Table I, we present the ratio $\tau(z) \equiv T_{z}(z) / T_{o b j}$ at $z=3.91,1.43$ and 1.55 , for the four combinations of model parameters $c$ and $\Omega_{m 0}$ of the fit values with $1 \sigma$ uncertainty. Obviously, $T_{z}(z)>T_{o b j}$ holds at $z=1.43$ and 1.55, whereas $T_{z}(z)<T_{o b j}$ at $z=3.91$. The old quasar APM $08279+5255$ at $z=3.91$ cannot be accommodated. In the other two cases with prior $h=0.72 \pm 0.08$ and $0.64 \leq h \leq 0.80$, we present the similar contents in Tables $\Pi$ and $\amalg$ respectively. As mentioned above, $T_{o b j}$ is proportional to the Hubble constant $H_{0}$. For the lower bound $h=0.64$, the dimensionless age parameter of the OHRO at $z=3.91,1.43$ and 1.55 are $T_{o b j}=0.131,0.262$ and 0.229 respectively. From Tables II and III] even for the lower bound of $T_{o b j}$, we find again that the old quasar APM $08279+5255$ at $z=3.91$ cannot be accommodated in these two cases.

\begin{tabular}{c|c|c|c}
\hline \hline$\left(c, \Omega_{m 0}\right)$ & $\tau(3.91)$ & $\tau(1.43)$ & $\tau(1.55)$ \\
\hline$(0.72,0.26)$ & 0.904 & 1.257 & 1.342 \\
$(1.14,0.26)$ & 0.892 & 1.217 & 1.302 \\
$(0.72,0.32)$ & 0.817 & 1.141 & 1.217 \\
$(1.14,0.32)$ & 0.808 & 1.112 & 1.188 \\
\hline \hline
\end{tabular}

TABLE II: The same as in Table $[$ but for the case of SNIa+CMB+LSS with prior $h=0.72 \pm 0.08$.

\begin{tabular}{c|c|c|c}
\hline \hline$\left(c, \Omega_{m 0}\right)$ & $\tau(3.91)$ & $\tau(1.43)$ & $\tau(1.55)$ \\
\hline$(0.69,0.26)$ & 0.905 & 1.260 & 1.345 \\
$(0.93,0.26)$ & 0.898 & 1.236 & 1.321 \\
$(0.69,0.31)$ & 0.830 & 1.161 & 1.238 \\
$(0.93,0.31)$ & 0.825 & 1.142 & 1.219 \\
\hline \hline
\end{tabular}

TABLE III: The same as in Table \ but for the case of SNIa+CMB+LSS with prior $0.64 \leq h \leq 0.80$.

Let us examine this age problem from another perspective. Following [50], we keep the model parameters $c$ and $\Omega_{m 0}$ free, and find out the parameter space which can accommodate these three OHRO by means of plotting the contours of the dimensionless age parameter. We scan the parameters in the ranges $0<\Omega_{m 0} \leq 1$ and $0<c \leq 4$. Note that $c$ and $\Omega_{m 0}$ cannot be zero in order to avoid divergence in Eqs. (12) and (5) with Eq. (8). And then, we obtain three contours $T_{z}(3.91)=T_{o b j}(3.91), T_{z}(1.43)=T_{o b j}(1.43)$ and $T_{z}(1.55)=T_{o b j}(1.55)$. As mentioned above, $T_{o b j}$ is proportional to the Hubble constant $H_{0}$ whereas $T_{z}(z)$ is independent of $H_{0}$. Thus, we take $h=0.64$, which is the lower bound of the final result $h=0.72 \pm 0.08$ of Freedman et al. 64]. We present these contours in Fig. 1. The allowed parameter spaces are the left regions of these contours, as indicated by the arrows. The WMAP3 bound $\Omega_{m 0}=0.268 \pm 0.018$ [6] is also indicated by two short-dashed lines. It is easy to see that the OHRO at $z=1.43$ and 1.55 can be accommodated. However, the OHRO at $z=3.91$ cannot be accommodated, since the allowed parameter space are out of the WMAP3 bound. Even when we use instead the loosest and model-independent cluster estimate $\Omega_{m 0}=0.3 \pm 0.1$ [63] which is indicated by two long-dashed lines, the allowed parameter space becomes very narrow for the OHRO at $z=3.91$. This narrowed parameter space $0.2 \leq \Omega_{m 0} \lesssim 0.22$, however, can also be ruled out by the combined constraints from the latest SNe Ia, galaxy clustering and 
CMB anisotropy [4]. Therefore, the old quasar APM 08279+5255 at $z=3.91$ cannot be accommodated in the original holographic dark energy model. The age problem also exists, like in the other dark energy models mentioned in Sec. I.

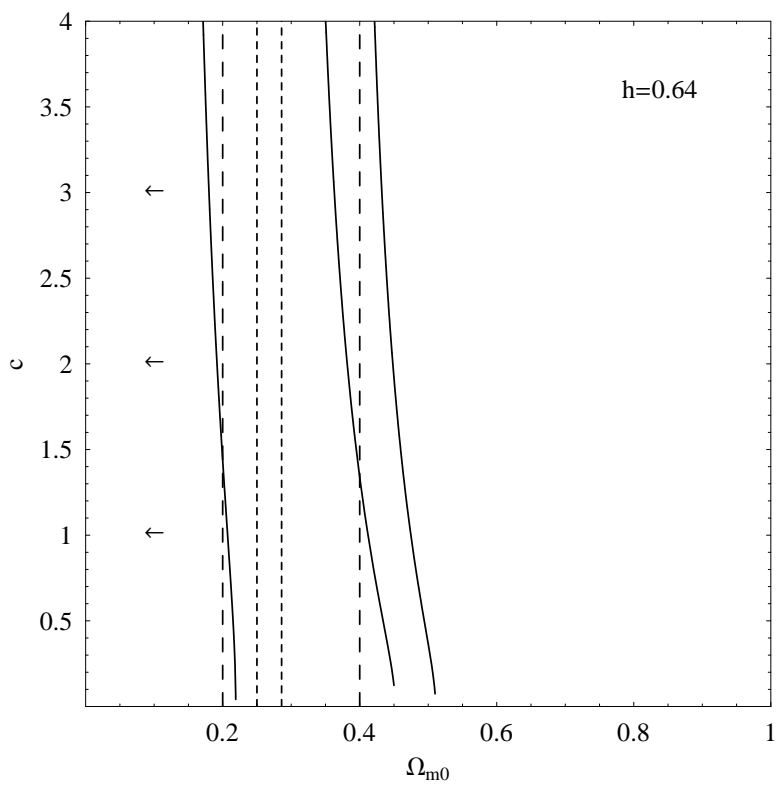

FIG. 1: The three solid lines are, from left to right, contours $T_{z}(3.91)=T_{o b j}(3.91), T_{z}(1.43)=T_{o b j}(1.43)$ and $T_{z}(1.55)=T_{o b j}(1.55)$. The WMAP3 bound $\Omega_{m 0}=0.268 \pm 0.018[\underline{6}]$ is indicated by two short-dashed lines. The model-independent cluster estimate $\Omega_{m 0}=0.3 \pm 0.1[63]$ is indicated by two long-dashed lines. The allowed parameter spaces are the left regions of these contours, as indicated by the arrows. In which, we have used the reduced Hubble constant $h=0.64$.

\section{ALLEVIATING THE AGE PROBLEM BY LOWER HUBBLE CONSTANT}

To solve the age problem, one way is to decrease the Hubble constant. This can be seen from Eq. (6). As mentioned above, $T_{z}(z)$ is independent of the Hubble constant $H_{0}$, whereas $T_{o b j}$ is proportional to the Hubble constant $H_{0}$. The lower $H_{0}$, the smaller $T_{o b j}$ is. Therefore, the condition Eq. (6) can be satisfied more easily. In the previous analysis, the reduced Hubble constant $h=0.72 \pm 0.08$ of Freedman et al. [64] has been used extensively. However, in the recent years, it is argued that there is systematic bias in the result of Freedman et al. 64]. Sandage and collaborators advocate a lower Hubble constant in a series of works [65, 66, 67, 68, 69]. Their final result reads $h=0.623 \pm 0.063$ [69].

At first, we take the central value of the result of Sandage et al. 69], namely $h=0.623$. We present the contours $T_{z}(3.91)=T_{o b j}(3.91), T_{z}(1.43)=T_{o b j}(1.43)$ and $T_{z}(1.55)=T_{o b j}(1.55)$ in Fig. 2. Obviously, the situation is similar to the case $h=0.64$ considered in the previous section, although there are some slight improvements. The OHRO at $z=1.43$ and 1.55 are easily accommodated. However, the old quasar APM $08279+5255$ at $z=3.91$ still cannot be accommodated in the original holographic dark energy model. This is mainly because the difference between $h=0.623$ and $h=0.64$ is too small.

Then, we consider the lower bound of the result of Sandage et al. [69], i.e. $h=0.56$. The corresponding contours are presented in Fig. 3. It is easy to see that the situation is improved significantly. The allowed 
parameter space for the OHRO at $z=3.91$ is fully consistent with the loosest model-independent cluster estimate $\Omega_{m 0}=0.3 \pm 0.1\left[63\right.$, the tighter WMAP3 bound $\Omega_{m 0}=0.268 \pm 0.018$ [6], and the combined constraints from the latest SNe Ia, galaxy clustering and CMB anisotropy [43]. In this case, all the three OHRO at $z=3.91,1.43$ and 1.55 can be accommodated in the original holographic dark energy model. The age problem disappears.

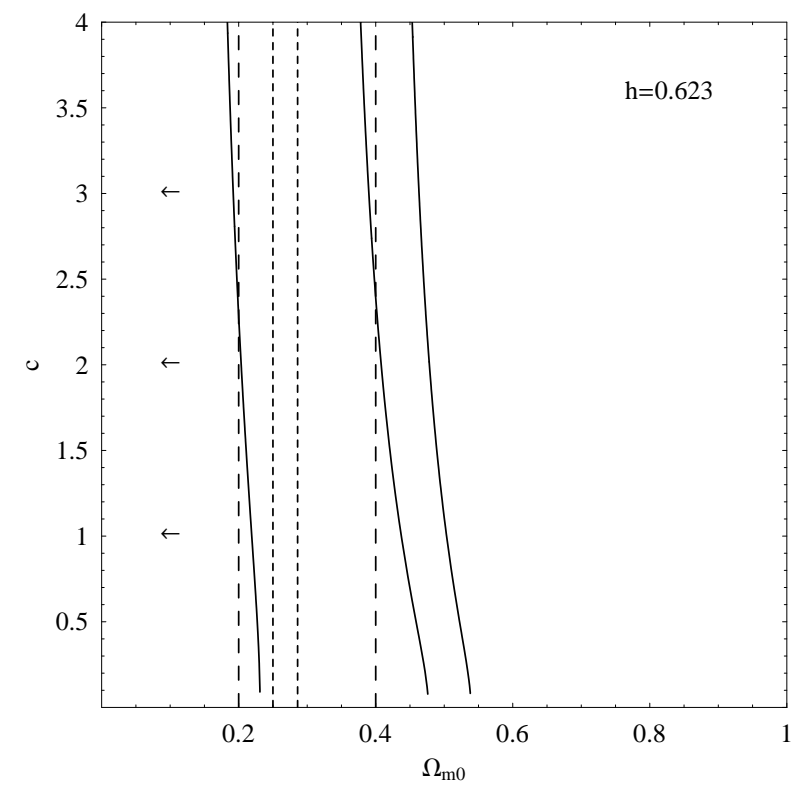

FIG. 2: The same as in Fig. 1 except for $h=0.623$.

\section{CONCLUDING REMARKS}

Actually, in addition to the three OHRO considered in this work, there are other OHRO in the literature, for instance, the 4.0 Gyr old radio galaxy 3C 65 at $z=1.175$ [70], and the high redshift quasar B1422+231 at $z=3.62$ whose best fit age is $1.5 \mathrm{Gyr}$ with a lower bound of $1.3 \mathrm{Gyr}$ [71]. However, they cannot be used to constrain the model parameters as restrictive as the previous three OHRO. Thus, we do not consider them in this work.

In this note, we test the original holographic dark energy model with some old high redshift objects. The main idea is very simple: the universe cannot be younger than its constituents. We find that the original holographic dark energy model can be ruled out, unless a lower Hubble constant is taken.

In fact, as mentioned in Sec. I the old quasar APM $08279+5255$ at $z=3.91$ cannot be accommodated in many dark energy models (including the $\Lambda \mathrm{CDM}$ model). The common way out is to use a lower Hubble constant instead. This hints that the extensively used $h=0.72 \pm 0.08$ of Freedman et al. 64 may have systematic bias. The ages of OHRO tend to a lower Hubble constant, say, the one recently advocated by Sandage and collaborators.

In the literature, there are still some debates on the value of the Hubble constant. After the final result $h=0.72 \pm 0.08$ of Freedman et al. 64], many authors argue for a lower Hubble constant, for instance, $h=0.68 \pm 0.07$ at $2 \sigma$ uncertainty in 72]. By now, the final result $h=0.623 \pm 0.063$ of Sandage and collaborators [69] attracted more and more attentions. We consider that it is important to take this into account when one tries to constrain the cosmological model parameters. The age problem in dark energy models supports this argument strongly. 
It is worth noting that the results in this work are obtained in the original holographic dark energy model. In fact, there are many modified holographic dark energy models in the literature, such as the nonsaturated holographic dark energy [73], the holographic dark energy model with variable $G_{N}$ [74], the interacting holographic dark energy model [75, 76], and so on. The conclusions of this work might be changed in these models. For instance, in the interacting holographic dark energy model, it is shown that for a fixed $c$, the holographic dark energy starts to be effective earlier and consequently the universe experiences the accelerated expansion earlier when the interaction is larger [76]. In this case, the universe can have a longer age. Therefore, it is of interest to examine the age problem in these modified holographic dark energy models in the future works.

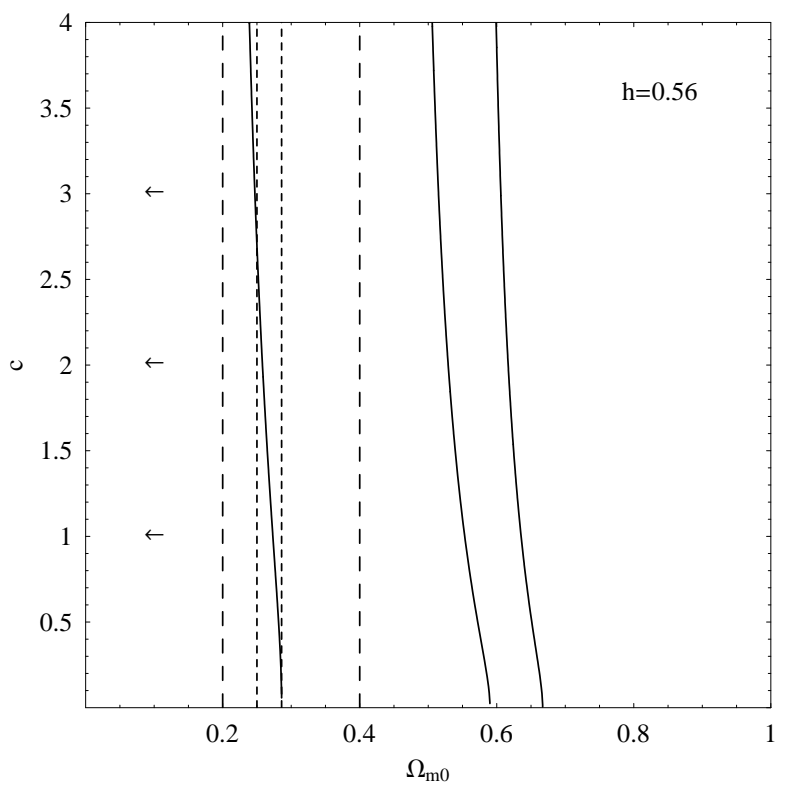

FIG. 3: The same as in Fig. 1. except for $h=0.56$.

\section{ACKNOWLEDGMENTS}

We are grateful to Prof. Rong-Gen Cai, Prof. Miao Li and Prof. Bin Wang for helpful discussions. We also thank Shi-Chao Tang, Jian Hu, Xin Liu, Yue Shen, Lin Lin, Sumin Tang, Jing Jin, Wei-Ke Xiao, Feng-Yun Rao, Nan Liang, Rong-Jia Yang, Jian Wang, Yuan Liu, and Xin Zhang, Zong-Kuan Guo, Qing-Guo Huang, Hui Li, Meng Su for kind help and discussions. The major computations of this work were completed during the period of the International Conference on Astrophysics of Compact Objects, Huangshan, China, July 2007. We acknowledge partial funding support from China Postdoctoral Science Foundation, and by the Ministry of Education of China, Directional Research Project of the Chinese Academy of Sciences under project No. KJCX2-YW-T03, and by the National Natural Science Foundation of China under project No. 10521001.

[1] P. J. E. Peebles and B. Ratra, Rev. Mod. Phys. 75, 559 (2003) astro-ph/0207347;

T. Padmanabhan, Phys. Rept. 380, 235 (2003) hep-th/0212290; 
S. M. Carroll, astro-ph/0310342

R. Bean, S. Carroll and M. Trodden, astro-ph/0510059

V. Sahni and A. A. Starobinsky, Int. J. Mod. Phys. D 9, 373 (2000) astro-ph/9904398;

S. M. Carroll, Living Rev. Rel. 4, 1 (2001) astro-ph/0004075;

T. Padmanabhan, Curr. Sci. 88, 1057 (2005) astro-ph/0411044;

S. Weinberg, Rev. Mod. Phys. 61, 1 (1989);

S. Nobbenhuis, Found. Phys. 36, 613 (2006) gr-qc/0411093;

E. J. Copeland, M. Sami and S. Tsujikawa, Int. J. Mod. Phys. D 15, 1753 (2006) hep-th/0603057;

A. Albrecht et al., astro-ph/0609591.

R. Trotta and R. Bower, astro-ph/0607066

M. Kamionkowski, arXiv:0706.2986 [astro-ph];

B. Ratra and M. S. Vogeley, arXiv:0706.1565 [astro-ph];

E. V. Linder, arXiv:0705.4102 [astro-ph].

[2] A. G. Riess et al. [Supernova Search Team Collaboration], Astron. J. 116, 1009 (1998) astro-ph/9805201; S. Perlmutter et al. [Supernova Cosmology Project Collaboration], Astrophys. J. 517, 565 (1999) astro-ph/9812133;

J. L. Tonry et al. [Supernova Search Team Collaboration], Astrophys. J. 594, 1 (2003) astro-ph/0305008; R. A. Knop et al. [Supernova Cosmology Project Collaboration], Astrophys. J. 598, 102 (2003) astro-ph/0309368;

A. G. Riess et al. [Supernova Search Team Collaboration], Astrophys. J. 607, 665 (2004) astro-ph/0402512.

[3] A. G. Riess et al. [Supernova Search Team Collaboration], astro-ph/0611572

The numerical data of the full sample are available at

http://braeburn.pha.jhu.edu/ ariess/R06 or upon request to ariess@stsci.edu

[4] P. Astier et al. [SNLS Collaboration], Astron. Astrophys. 447, 31 (2006) astro-ph/0510447;

J. D. Neill et al. [SNLS Collaboration], astro-ph/0605148

[5] C. L. Bennett et al. [WMAP Collaboration], Astrophys. J. Suppl. 148, 1 (2003) astro-ph/0302207;

D. N. Spergel et al. [WMAP Collaboration], Astrophys. J. Suppl. 148175 (2003) astro-ph/0302209].

[6] D. N. Spergel et al. [WMAP Collaboration], astro-ph/0603449;

L. Page et al. [WMAP Collaboration], astro-ph/0603450

G. Hinshaw et al. [WMAP Collaboration], astro-ph/0603451

N. Jarosik et al. [WMAP Collaboration], astro-ph/0603452.

[7] M. Tegmark et al. [SDSS Collaboration], Phys. Rev. D 69, 103501 (2004) astro-ph/0310723;

M. Tegmark et al. [SDSS Collaboration], Astrophys. J. 606, 702 (2004) astro-ph/0310725;

U. Seljak et al., Phys. Rev. D 71, 103515 (2005) astro-ph/0407372;

J. K. Adelman-McCarthy et al. [SDSS Collaboration], Astrophys. J. Suppl. 162, 38 (2006) astro-ph/0507711];

K. Abazajian et al. [SDSS Collaboration], astro-ph/0410239; astro-ph/0403325; astro-ph/0305492.

M. Tegmark et al. [SDSS Collaboration], Phys. Rev. D 74, 123507 (2006) astro-ph/0608632].

[8] S. W. Allen, R. W. Schmidt, H. Ebeling, A. C. Fabian and L. van Speybroeck, Mon. Not. Roy. Astron. Soc. 353, 457 (2004) astro-ph/0405340;

S. W. Allen, D. A. Rapetti, R. W. Schmidt, H. Ebeling, G. Morris and A. C. Fabian, arXiv:0706.0033 [astro-ph].

[9] W. M. Wood-Vasey et al. [ESSENCE Collaboration], astro-ph/0701041

G. Miknaitis et al. [ESSENCE Collaboration], astro-ph/0701043.

[10] R. R. Caldwell, R. Dave and P. J. Steinhardt, Phys. Rev. Lett. 80, 1582 (1998) astro-ph/9708069;

C. Wetterich, Nucl. Phys. B 302, 668 (1988);

P. J. E. Peebles and B. Ratra, Astrophys. J. 325, L17 (1988);

B. Ratra and P. J. E. Peebles, Phys. Rev. D 37, 3406 (1988).

[11] P. J. Steinhardt, L. M. Wang and I. Zlatev, Phys. Rev. D 59, 123504 (1999) astro-ph/9812313;

I. Zlatev and P. J. Steinhardt, Phys. Lett. B 459, 570 (1999) astro-ph/9906481.

[12] R. R. Caldwell, Phys. Lett. B 545, 23 (2002) astro-ph/9908168.

[13] R. R. Caldwell, M. Kamionkowski and N. N. Weinberg, Phys. Rev. Lett. 91, 071301 (2003) astro-ph/0302506.

[14] S. M. Carroll, M. Hoffman and M. Trodden, Phys. Rev. D 68, 023509 (2003) astro-ph/0301273;

J. M. Cline, S. Y. Jeon and G. D. Moore, Phys. Rev. D 70, 043543 (2004) hep-ph/0311312.

[15] C. Armendariz-Picon, V. Mukhanov and P. J. Steinhardt, Phys. Rev. Lett. 85, 4438 (2000) 
astro-ph/0004134;

C. Armendariz-Picon, V. Mukhanov and P. J. Steinhardt, Phys. Rev. D 63, 103510 (2001) astro-ph/0006373;

T. Chiba, T. Okabe and M. Yamaguchi, Phys. Rev. D 62, 023511 (2000) astro-ph/9912463;

M. Malquarti, E. J. Copeland and A. R. Liddle, Phys. Rev. D 68, 023512 (2003) astro-ph/0304277;

M. Malquarti, E. J. Copeland, A. R. Liddle and M. Trodden, Phys. Rev. D 67, 123503 (2003) astro-ph/0302279;

H. Wei and R. G. Cai, Phys. Rev. D 71, 043504 (2005) hep-th/0412045].

[16] B. Feng, X. L. Wang and X. M. Zhang, Phys. Lett. B 607, 35 (2005) astro-ph/0404224].

[17] Z. K. Guo, Y. S. Piao, X. M. Zhang and Y. Z. Zhang, Phys. Lett. B 608, 177 (2005) astro-ph/0410654.

[18] H. Wei and R. G. Cai, Phys. Lett. B 634, 9 (2006) astro-ph/0512018.

Z. K. Guo, Y. S. Piao, X. M. Zhang and Y. Z. Zhang, Phys. Rev. D 74, 127304 (2006) astro-ph/0608165;

X. F. Zhang, H. Li, Y. S. Piao and X. M. Zhang, Mod. Phys. Lett. A 21, 231 (2006) astro-ph/0501652.

M. Z. Li, B. Feng and X. M. Zhang, JCAP 0512, 002 (2005) hep-ph/0503268;

X. F. Zhang and T. T. Qiu, Phys. Lett. B 642, 187 (2006) astro-ph/0603824;

Y. F. Cai, H. Li, Y. S. Piao and X. M. Zhang, Phys. Lett. B 646, 141 (2007) gr-qc/0609039;

Y. F. Cai, M. Z. Li, J. X. Lu, Y. S. Piao, T. T. Qiu and X. M. Zhang, Phys. Lett. B 651, 1 (2007) hep-th/0701016;

Y. F. Cai, T. T. Qiu, Y. S. Piao, M. Z. Li and X. M. Zhang, arXiv:0704.1090 [gr-qc].

[19] R. Lazkoz and G. Leon, Phys. Lett. B 638, 303 (2006) astro-ph/0602590;

R. Lazkoz, G. Leon and I. Quiros, Phys. Lett. B 649, 103 (2007) astro-ph/0701353;

M. Alimohammadi, arXiv:0706.1360 [gr-qc].

[20] H. Wei, R. G. Cai and D. F. Zeng, Class. Quant. Grav. 22, 3189 (2005) hep-th/0501160;

H. Wei and R. G. Cai, Phys. Rev. D 72, 123507 (2005) astro-ph/0509328;

M. Alimohammadi and H. Mohseni Sadjadi, Phys. Rev. D 73, 083527 (2006) hep-th/0602268;

W. Zhao and Y. Zhang, Phys. Rev. D 73, 123509 (2006) astro-ph/0604460;

H. Wei, N. N. Tang and S. N. Zhang, Phys. Rev. D 75, 043009 (2007) astro-ph/0612746;

H. Wei and S. N. Zhang, arXiv:0705.4002 [gr-qc];

W. Zhao, arXiv:0706.2211 [astro-ph].

[21] G. 't Hooft, gr-qc/9310026.

L. Susskind, J. Math. Phys. 36, 6377 (1995) hep-th/9409089.

[22] R. Bousso, Rev. Mod. Phys. 74, 825 (2002) hep-th/0203101].

[23] J. D. Bekenstein, Phys. Rev. D 7 (1973) 2333;

J. D. Bekenstein, Phys. Rev. D 9, 3292 (1974);

J. D. Bekenstein, Phys. Rev. D 23, 287 (1981);

J. D. Bekenstein, Phys. Rev. D 49, 1912 (1994) gr-qc/9307035].

[24] S. W. Hawking, Commun. Math. Phys. 43, 199 (1975) [Erratum-ibid. 46, 206 (1976)];

S. W. Hawking, Phys. Rev. D 13, 191 (1976).

[25] A. G. Cohen, D. B. Kaplan and A. E. Nelson, Phys. Rev. Lett. 82, 4971 (1999) hep-th/9803132.

[26] P. Horava and D. Minic, Phys. Rev. Lett. 85, 1610 (2000) hep-th/0001145;

S. D. Thomas, Phys. Rev. Lett. 89, 081301 (2002).

[27] S. D. H. Hsu, Phys. Lett. B 594, 13 (2004) hep-th/0403052.

[28] W. Fischler and L. Susskind, hep-th/9806039

R. Bousso, JHEP 9907, 004 (1999) hep-th/9905177.

[29] M. Li, Phys. Lett. B 603, 1 (2004) hep-th/0403127.

[30] Q. G. Huang and M. Li, JCAP 0408, 013 (2004) astro-ph/0404229];

K. Ke and M. Li, Phys. Lett. B 606, 173 (2005) hep-th/0407056;

Q. G. Huang and M. Li, JCAP 0503, 001 (2005) hep-th/0410095;

B. Chen, M. Li and Y. Wang, Nucl. Phys. B 774, 256 (2007) astro-ph/0611623.

[31] M. Ito, Europhys. Lett. 71, 712 (2005) hep-th/0405281;

P. X. Wu and H. W. Yu, Int. J. Mod. Phys. D 14, 1873 (2005) gr-qc/0509036;

B. Guberina, R. Horvat and H. Nikolic, Phys. Rev. D 72, 125011 (2005) astro-ph/0507666;

B. Guberina, R. Horvat and H. Nikolic, Phys. Rev. D 72, 125011 (2005) astro-ph/0507666;

J. P. Beltran Almeida and J. G. Pereira, Phys. Lett. B 636, 75 (2006) gr-qc/0602103;

M. S. Berger and H. Shojaei, Phys. Rev. D 74, 043530 (2006) astro-ph/0606408; 
H. M. Sadjadi and M. Honardoost, Phys. Lett. B 647, 231 (2007) gr-qc/0609076;

H. M. Sadjadi, JCAP 0702, 026 (2007) gr-qc/0701074;

F. Simpson, JCAP 0703, 016 (2007) astro-ph/0609755;

B. Guberina, R. Horvat and H. Nikolic, JCAP 0701, 012 (2007) astro-ph/0611299;

P. Diaz, M. A. Per and A. Segui, arXiv:0704.1637 [hep-th].

[32] X. Zhang, Int. J. Mod. Phys. D 14, 1597 (2005) astro-ph/0504586;

X. Zhang, Phys. Lett. B 648, 1 (2007) astro-ph/0604484;

X. Zhang, Phys. Rev. D 74, 103505 (2006) astro-ph/0609699];

M. R. Setare, J. Zhang and X. Zhang, JCAP 0703, 007 (2007) gr-qc/0611084;

J. F. Zhang, X. Zhang and H. Y. Liu, astro-ph/0612642,

J. Zhang, X. Zhang and H. Liu, arXiv:0705.4145 [astro-ph];

J. Zhang, X. Zhang and H. Liu, arXiv:0706.1185 [astro-ph].

[33] W. Zimdahl, Int. J. Mod. Phys. D 14, 2319 (2005) gr-qc/0505056;

D. Pavon and W. Zimdahl, AIP Conf. Proc. 841, 356 (2006) hep-th/0511053;

W. Zimdahl and D. Pavon, astro-ph/0606555;

D. Pavon, J. Phys. A 40, 6865 (2007) gr-qc/0610008;

N. Banerjee and D. Pavon, Phys. Lett. B 647, 477 (2007) gr-qc/0702110.

[34] Y. G. Gong, Phys. Rev. D 70, 064029 (2004) hep-th/0404030;

Y. G. Gong and Y. Z. Zhang, Class. Quant. Grav. 22, 4895 (2005) hep-th/0505175.

[35] Y. S. Myung, Phys. Lett. B 626, 1 (2005) hep-th/0502128;

H. Kim, H. W. Lee and Y. S. Myung, Phys. Lett. B 632, 605 (2006) gr-qc/0509040;

Y. S. Myung, Phys. Lett. B 649, 247 (2007) gr-qc/0702032;

K. Y. Kim, H. W. Lee and Y. S. Myung, arXiv:0706.2444 [gr-qc];

Y. S. Myung, arXiv:0706.3757 [gr-qc].

[36] B. Hu and Y. Ling, Phys. Rev. D 73, 123510 (2006) hep-th/0601093;

H. Li, Z. K. Guo and Y. Z. Zhang, Int. J. Mod. Phys. D 15, 869 (2006) astro-ph/0602521.

[37] M. R. Setare and S. Shafei, JCAP 0609, 011 (2006) gr-qc/0606103;

M. R. Setare, Phys. Lett. B 642, 1 (2006) hep-th/0609069;

M. R. Setare, hep-th/0609104.

M. R. Setare, Phys. Lett. B 644, 99 (2007) hep-th/0610190;

M. R. Setare, Eur. Phys. J. C 50, 991 (2007) hep-th/0701085];

M. R. Setare, JCAP 0701, 023 (2007) hep-th/0701242;

M. R. Setare and E. C. Vagenas, arXiv:0704.2070 [hep-th];

M. R. Setare, Phys. Lett. B 648, 329 (2007) arXiv:0704.3679;

M. R. Setare, arXiv:0705.3517 [hep-th].

[38] Q. G. Huang and Y. G. Gong, JCAP 0408, 006 (2004) astro-ph/0403590;

B. Wang, Y. G. Gong and R. K. Su, Phys. Lett. B 605, 9 (2005) hep-th/0408032;

Y. G. Gong, B. Wang and Y. Z. Zhang, Phys. Rev. D 72, 043510 (2005) hep-th/0412218;

Y. G. Gong, Int. J. Mod. Phys. D 14, 599 (2005) astro-ph/0401207;

X. Zhang and F. Q. Wu, Phys. Rev. D 72, 043524 (2005) astro-ph/0506310;

Q. Wu, Y. Gong, A. Wang and J. S. Alcaniz, arXiv:0705.1006 [astro-ph];

C. Feng, B. Wang, Y. Gong and R. K. Su, arXiv:0706.4033 [astro-ph].

[39] J. Y. Shen, B. Wang, E. Abdalla and R. K. Su, Phys. Lett. B 609, 200 (2005) hep-th/0412227;

H. C. Kao, W. L. Lee and F. L. Lin, Phys. Rev. D 71, 123518 (2005) astro-ph/0501487.

[40] Z. Chang, F. Q. Wu and X. Zhang, Phys. Lett. B 633, 14 (2006) astro-ph/0509531.

[41] Z. L. Yi and T. J. Zhang, Mod. Phys. Lett. A 22, 41 (2007) astro-ph/0605596.

[42] H. Zhang, W. Zhong, Z. Zhu and S. He, arXiv:0705.4409 [astro-ph].

[43] X. Zhang and F. Q. Wu, Phys. Rev. D 76, 023502 (2007) astro-ph/0701405.

[44] J. S. Alcaniz and J. A. S. Lima, Astrophys. J. 521, L87 (1999) astro-ph/9902298].

[45] E. W. Kolb and M. S. Turner, The Early Universe, Addison-Wesley Publishing Company, USA (1990) [in the series Front. Phys. 69, 1 (1990)].

[46] J. Dunlop et al., Nature 381, 581 (1996).

[47] H. Spinrad et al., Astrophys. J. 484, 581 (1997).

[48] J. Dunlop, in The Most Distant Radio Galaxies, edited by H. J. A. Rottgering, P. Best and M. D. Lehnert, 
Kluwer, Dordrecht (1999), p. 71.

[49] J. A. S. Lima and J. S. Alcaniz, Mon. Not. Roy. Astron. Soc. 317, 893 (2000) astro-ph/0005441.

[50] D. Jain and A. Dev, Phys. Lett. B 633, 436 (2006) astro-ph/0509212.

[51] G. Hasinger, N. Schartel and S. Komossa, Astrophys. J. 573, L77 (2002) astro-ph/0207005.

[52] S. Komossa and G. Hasinger, astro-ph/0207321.

[53] A. Friaca, J. Alcaniz and J. A. S. Lima, Mon. Not. Roy. Astron. Soc. 362, 1295 (2005) astro-ph/0504031.

[54] J. S. Alcaniz, J. A. S. Lima and J. V. Cunha, Mon. Not. Roy. Astron. Soc. 340, L39 (2003) astro-ph/0301226.

[55] J. S. Alcaniz, D. Jain and A. Dev, Phys. Rev. D 67, 043514 (2003) astro-ph/0210476].

[56] J. V. Cunha and R. C. Santos, Int. J. Mod. Phys. D 13, 1321 (2004) astro-ph/0402169; J. F. Jesus, astro-ph/0603142

[57] M. A. Dantas, J. S. Alcaniz, D. Jain and A. Dev, Astron. Astrophys. 467, 421 (2007) astro-ph/0607060.

[58] S. Capozziello, P. K. S. Dunsby, E. Piedipalumbo and C. Rubano, arXiv:0706.2615 [astro-ph].

[59] M. S. Movahed, S. Baghram and S. Rahvar, arXiv:0705.0889 [astro-ph].

[60] M. S. Movahed, M. Farhang and S. Rahvar, astro-ph/0701339.

M. S. Movahed and S. Ghassemi, arXiv:0705.3894 [astro-ph].

[61] N. Pires, Z. H. Zhu and J. S. Alcaniz, Phys. Rev. D 73, 123530 (2006) astro-ph/0606689.

[62] S. Rahvar and M. S. Movahed, Phys. Rev. D 75, 023512 (2007) astro-ph/0604206.

[63] R. Carlberg, H. K. C. Yee, E. Ellingson, R. Abraham, P. Gravel, S. Morris and C. J. Pritchet, Astrophys. J. 462, 32 (1996) astro-ph/9509034;

A. Dekel, D. Burstein and S. White, in Critical Dialogues in Cosmology, edited by N. Turok, World Scientific, Singapore (1997).

[64] W. L. Freedman et al., Astrophys. J. 553, 47 (2001) astro-ph/0012376.

[65] G. A. Tammann, A. Sandage and B. Reindl, Astron. Astrophys. 404, 423 (2003) astro-ph/0303378.

[66] A. Sandage, G. A. Tammann and B. Reindl, Astron. Astrophys. 424, 43 (2004) astro-ph/0402424.

[67] B. Reindl, G. A. Tammann, A. Sandage and A. Saha, Astrophys. J. 624, 532 (2005) astro-ph/0501664].

[68] A. Saha, F. Thim, G. A. Tammann, B. Reindl, and A. Sandage Astrophys. J. Suppl. 165, 108 (2006) astro-ph/0602572.

[69] A. Sandage, G. A. Tammann, A. Saha, B. Reindl, F. D. Macchetto and N. Panagia, Astrophys. J. 653, 843 (2006) astro-ph/0603647.

[70] A. Stockton, M. Kellogg and S. E. Ridgway, Astrophys. J. 443, L69 (1995).

[71] Y. Yoshii, T. Tsujimoto and K. Kawara, Astrophys. J. 507, L113 (1998) astro-ph/9809047.

[72] J. R. I. Gott, M. S. Vogeley, S. Podariu and B. Ratra, Astrophys. J. 549, 1 (2001) astro-ph/0006103;

G. Chen, J. R. I. Gott and B. Ratra, Publ. Astron. Soc. Pac. 115, 1269 (2003) astro-ph/0308099.

[73] B. Guberina, R. Horvat and H. Nikolic, JCAP 0701, 012 (2007) astro-ph/0611299.

[74] B. Guberina, R. Horvat and H. Nikolic, Phys. Lett. B 636, 80 (2006) astro-ph/0601598.

[75] R. Horvat, Phys. Rev. D 70, 087301 (2004) astro-ph/0404204];

D. Pavon and W. Zimdahl, Phys. Lett. B 628, 206 (2005) gr-qc/0505020].

[76] B. Wang, Y. G. Gong and E. Abdalla, Phys. Lett. B 624, 141 (2005) hep-th/0506069. 